\title{
Exoresection via partial lamellar sclerouvectomy approach for uveal tumors:A successful performance by a novice surgeon
}

This article was published in the following Dove Press journal:

Clinical Ophthalmology

28 January 2010

Number of times this article has been viewed

\section{Rengin Aslıhan Kurt \\ Kaan Gündüz}

Department of Ophthalmology, Ankara University Faculty of Medicine, Ankara, Turkey
Correspondence: Kaan Gündüz Mesa Koza Plaza 24/I8, Küpe Sok, GOP 06700, Ankara, Turkey Tel +90 5326337997

Email eyemd@ada.net.tr
Purpose: To evaluate the results and complications of exoresection via a partial lamellar sclerouvectomy (PLSU) procedure for uveal tumors performed by a novice surgeon without help from an experienced surgeon.

Methods: Medical records of 22 patients who underwent exoresection for uveal tumors between February 1999 and January 2009 were evaluated retrospectively. Exoresection was considered for tumors with their epicenter in the iris or in the ciliary body.

Results: Twenty-two patients with a mean age of 45.9 years (range: 19-72 years) were included in this study. The histopathologic diagnosis was uveal malignant melanoma in 16 patients, iridociliary nevus in 2 patients, iris nevus in 2 patients, and iridociliary melanocytoma in 2 patients. Postoperative complications included cataract in 11 patients $(50 \%)$, scleral thinning in 4 patients $(18 \%)$, vitreous hemorrhage in 2 patients $(9 \%)$, hyphema in 2 patients $(9 \%)$, secondary glaucoma in 2 patients $(9 \%)$, iridodialysis in 1 patient (4.5\%), bullous keratopathy in 1 patient $(4.5 \%)$, and posterior synechiae in 1 patient (4.5\%). At a mean follow-up of 40.1 months (range: 1-98 months), there were no recurrences or metastatic events.

Conclusions: Exoresection seems to be an effective treatment option in selected cases of iridociliary and ciliary body tumors. Novice surgeons with limited training in PLSU should not discourage themselves from doing this type of surgery. Several postoperative complications inevitably occur and require frequent patient monitoring.

Keywords: uveal melanoma, uveal nevus, iris, ciliary body, choroid, iris nevus, iris melanoma, ciliary nevus, ciliary melanoma, choroidal melanoma, ciliochoroidal melanoma, iridociliochoroidal melanoma, partial lamellar sclerouvectomy, exoresection

\section{Introduction}

The indications for surgical removal of uveal tumors are numerous. Removal is indicated for iridociliary tumors of indeterminate pathology that present suspicious features, such as pigment seeding, prominent vascularity, and increased thickness and size. ${ }^{1,2}$ It may not be possible to perform fine-needle aspiration biopsy (FNAB) of these tumors, especially if they are located in the iridocorneal angle. Moreover, different portions of the tumor may harbor different pathologies, so small FNAB samples may be misleading. ${ }^{3}$ Second, iridociliary tumors with documented growth, such as leiomyoma and melanocytoma, need to be surgically excised to restore intraocular structure and prevent complications such as lens subluxation and cataract. ${ }^{4}$ Growing necrotic iridociliary melanocytomas can cause increase in intraocular pressure (IOP); thus, removal is also necessary to achieve control of IOP and intraocular inflammation. ${ }^{5}$ Third, in developing countries, plaque radiotherapy or proton beam 
radiotherapy for iridociliary melanomas may not be available. Plaque radiotherapy requires custom-designed plaques, ${ }^{6,7}$ and proton beam radiotherapy requires complex instrumentation. Both treatments may be prohibitively expensive, if they are available at all. Surgical resection may provide an alternative means of tumor control for iris and certain iridociliary melanomas under these circumstances. Fourth, thick ciliary body and choroidal melanomas require high radiation doses to the tumor and surrounding tissues, resulting in severe radiation complications. Surgical resection is believed to be safer under these circumstances. ${ }^{8}$

For the reasons outlined above, the ocular oncologist should be familiar with the techniques of partial lamellar sclerouvectomy (PLSU) and be able to perform this surgery. It is generally believed that iridociliary tumors occupying less than three clock hours of pars plicata and choroidal melanomas less than $15 \mathrm{~mm}$ in base diameter are eligible for surgical exoresection. ${ }^{9-11}$ Although certain surgeons at worldwide recognized centers have gained wide experience with exoresection using PLSU, ${ }^{9-12}$ many oncology fellowship programs do not offer extensive hands-on experience with this technique. Thus, many novice surgeons after fellowship training with limited experience in exoresection as a first surgeon may be reluctant to start doing this type of surgery on their own. In this paper, we describe our use of PLSU technique in managing 22 cases of uveal tumors. We address the potential difficulties of performing the technique and describe common complications, as experienced by a novice surgeon $(\mathrm{KG})$ starting on his own without assistance of an experienced surgeon.

\section{Materials and methods}

We reviewed the clinical charts of 22 patients who underwent exoresection for uveal tumors between February 1999 and January 2009. Institutional Review Board approval was obtained for reviewing and publishing the results. All patients included in this study underwent complete ophthalmic examination, including visual acuity and IOP measurement, biomicroscopy, and dilated fundus examinations via indirect ophthalmoscopy. Tumor dimensions, including base diameter and thickness, were determined by indirect ophthalmoscopy, A and B mode ultrasonography, and ultrasound biomicroscopy, depending on tumor location. The decision for tumor removal was based on one of the following criteria: 1) Suspicious iris/iridocorneal angle/iridociliary tumors with pigment dispersion, prominent tumor vascularity, tumor base diameter $>3 \mathrm{~mm}$, and thickness $>2 \mathrm{~mm}$ on ultrasound biomicroscopy (UBM) or documented growth. 2) Iris, iridociliary, ciliochoroidal and iridociliochoroidal melanoma diagnosed by clinical examination, ultrasonography, UBM, and/or anterior segment/fundus photography. We performed exoresection in suspected melanoma cases where the epicenter of the tumor was in the iris or ciliary body with minimal extension into the adjacent choroid. 3) Patient desire to have the intraocular tumor removed rather than followed up with observation despite absence of compelling evidence for malignancy or growth.

Informed consent was obtained before surgery. The surgical procedure in all cases was performed by the same surgeon (KG) who after completing his oncology fellowship started doing this surgery on his own. The surgical technique has been described previously. ${ }^{8,9}$ Hypotensive general anesthesia was used to keep the systemic blood pressure low. A slightly reverse Trendelenburg positon was preferred to reduce bleeding. After a Williams lid speculum was inserted, a $270^{\circ}$ limbal peritomy was done. Three rectus muscles around the area of the tumor were hooked and isolated with $2 / 0$ silk sutures. If the tumor was under one of the rectus muscles, the overlying muscle was disinserted using a double-armed 6/0 vicryl suture. Using blunt dissection with Steven's scissors, the conjunctiva and Tenon's fascia were separated away from the globe in the quadrant of the tumor. Using bipolar diathermy, episcleral blood vessels were cauterized until the bare sclera was exposed.

After the eye was rotated to the side opposite the tumor, the margins of the tumor were localized by transillumination using a bright focal light on the side of the eye exactly opposite the tumor. The margins of the tumor on the sclera were marked with a marking pencil around the tumor shadow. Subsequently, the margins of the anteriorly hinged scleral flap positioned 3-4 mm outside this mark were marked on the sclera. The shape of the scleral flap was rectangular. With a \#57 beaver blade, a groove was created to an approximately $80 \%-90 \%$ thickness of the sclera, and the scleral flap was then developed and carried anteriorly to the corneoscleral limbus. If the blue color of the underlying choroid was not visible, the plane of dissection was gently deepened until it could be seen. Any bleeding from emissary vessels was controlled with bipolar cautery. If the tumor was in the iridocorneal angle, its shadow overlapped with the shadow of the pars plicata. In this case, the posterior margin of the tumor was marked at the posterior boundary of the pars plicata and lateral margins were marked according to the projected trajectory based on the contour of the iris tumor.

When the scleral flap was fully developed, transillumination was repeated. The margins of the tumor were outlined on the remaining inner scleral tissue with a marking pencil. A small circumferential incision using a $15^{\circ}$ microsurgical 
knife was then made on the inner scleral fibers about $3 \mathrm{~mm}$ outside the margins of the tumor. Then, Vannas scissors were used to complete the scleral incision until the uveal tract was exposed. The uveal tract was cauterized using bipolar cautery until blanching and a gray color change of the uveal tissue was achieved.

In uveal tumors involving the iris, a $15^{\circ}$ microsurgical knife was used to create a paracentesis port in the limbus approximately $90^{\circ}$ from the area of the main incision, and viscoelastic was injected into the anterior chamber. The anterior chamber was then entered using a $15^{\circ}$ microsurgical knife at the center of the flap area, and the corneoscleral incision was made laterally with Westcott scissors. For uveal tumors not involving the iris, the anterior chamber was not entered. In such cases, if there was marked bulging of the uveal tract, aspiration of approximately $1 \mathrm{~mL}$ of vitreous was achieved via a 22-gauge needle inserted into the central vitreous cavity through the pars plana. If there was no obvious bulging of the uvea, vitreous aspiration was omitted.

Subsequently, the uveal tumor was excised using Vannas scissors. The choroidal/ciliary part of the tumor was excised first, followed by excision of the iris part if the tumor had an iris component. The pupillary rim was spared, if possible. Care was taken to leave the underlying retina or, in the case of ciliary tumors, the nonpigmented ciliary pigment epithelium intact. The uveal tumor together with the overlying scleral fibers was carefully lifted off the surgical field using a "no-touch technique" to avoid spilling tumor cells into the surgical field. If vitreous loss occurred, vitreous was removed with a vitrectomy instrument. Before doing vitrectomy, three to five 9-0 nylon sutures were placed at the corners and the free margin of the rectangular scleral flap. Vitrectomy was subsequently done at the margins of the flap to prevent vitreous wick in the wound area. Following completion of the vitrectomy, the scleral flap was sutured to its original position with more 9-0 nylon sutures. Balanced salt solution was injected into the eye via the limbal paracentesis port to adjust the IOP, if necessary. Cellulose sponges were used to check to see if the wound was watertight. If there was any leak, more 9-0 nylon sutures were placed. Any previously detached rectus muscle was sutured to its original position/insertion, and the conjunctiva was reapproximated to the limbus with 7/0 vicryl sutures.

Adjunctive plaque radiotherapy with a $20 \mathrm{~mm}$ or $25 \mathrm{~mm}$ round Ruthenium-106 plaque was done 1 month after exoresection in uveal melanoma cases with predominant ciliary body involvement. We did not do plaque radiotherapy in iridociliary or iridocorneal melanomas where the epicenter of the tumor was in the iris with limited involvement of the adjacent ciliary body. We aimed to deliver an apex dose of $100 \mathrm{~Gy}$ to a $1 \mathrm{~mm}$ depth from the inner surface of the sclera during plaque radiotherapy.

\section{Results}

Of 22 patients who underwent exoresection for uveal tumors 15 were females $(68.2 \%)$ and 7 were males $(31.8 \%)$. The tumor was located in the right eye in 11 patients $(50 \%)$ and in the left eye in 11 patients $(50 \%)$. The mean age of the patients at the time of surgery was 45.9 years (range: 19-72 years).

The initial Snellen visual acuity ranged from hand motions to $10 / 10$. On the $\log$ MAR scale, initial visual acuity ranged from $0-1.9$ with a mean of $0.5 \log$ MAR. The mean preoperative IOP was $16.3 \mathrm{mmHg}$ (range: $11-34 \mathrm{mmHg}$ ). Although focal cataract and notching of the lens were noted in the lens overlying the tumor, none of the patients had significant cataracts necessitating extraction. Tumor base at the largest point ranged from $1.5 \mathrm{~mm}$ to $15.0 \mathrm{~mm}$ (mean: $7.5 \mathrm{~mm}$ ). Tumor thickness ranged from 0.9 to $7.0 \mathrm{~mm}$ (mean: $3.1 \mathrm{~mm}$ ). All tumors were pigmented. The tumor was located in the nasal quadrant in 10 eyes $(45.5 \%)$, in the temporal quadrant in 7 eyes $(32 \%)$, in the inferior quadrant in 4 eyes $(18 \%)$, and in the superior quadrant in 1 eye $(4.5 \%)$. The tumor was located in both the iris and ciliary body (iridociliary) in 11 eyes (50\%) (Figure 1a), in the iridocorneal angle in 6 eyes $(27 \%)$, in the ciliary body in 3 eyes $(14 \%)$, in the choroid and ciliary body (ciliochoroidal) in 1 eye $(4.5 \%)$, and in the iris, ciliary body and choroid in one eye $(4.5 \%)$ (Figure 2a)

The tumors were excised completely in all cases (Figures $1 \mathrm{~b}$, 2b, 2d, 3). Intraoperative complications included minor bleeding from the resection site, which occurred in almost all cases and was usually limited. Vitreous loss developed in 10 of the 20 eyes with iris and/or ciliary tumors and was treated with vitrectomy at the wound margins. The histopathologic diagnosis was uveal malignant melanoma in 16 eyes (iridocorneal angle melanoma in 6 eyes, iridociliary melanoma in 5 eyes, ciliary melanoma in 3 eyes, ciliochoroidal melanoma in 1 eye, iridociliochoroidal melanoma in 1 eye), iridociliary nevus in 4 patients, and iridociliary melanocytoma in 2 patients.

Postoperative complications included cataract in 11 patients $(50 \%)$ that was not present before surgery, scleral thinning in 4 patients (18\%) (Figure 3), nonresolving ( $>8$ weeks) vitreous hemorrhage in 2 patients $(9 \%)$, secondary glaucoma in 2 patients $(9 \%)$, hyphema in one patient $(4.5 \%)$, iridodialysis in 1 patient $(4.5 \%)$, bullous keratopathy in 1 patient $(4.5 \%)$, and posterior synechiae 


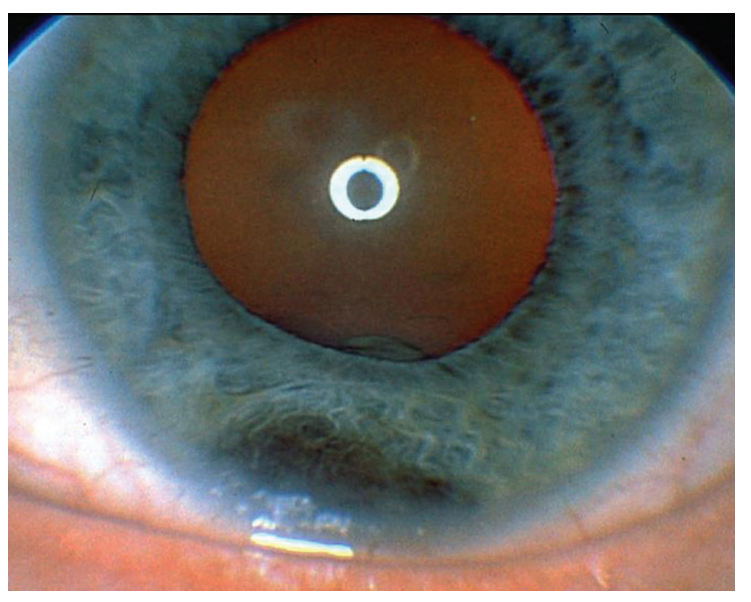

Figure I a Inferiorly located iridociliary melanoma.

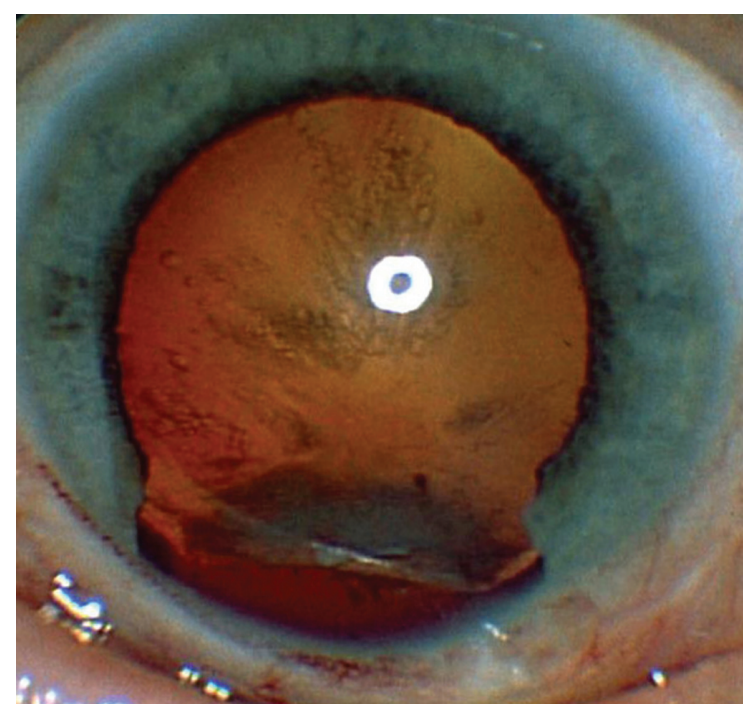

Figure Ib After exoresection via partial lamellar sclerouvectomy and complete tumor removal, notching of the lens induced by the tumor and posterior subcapsular cataract are seen.

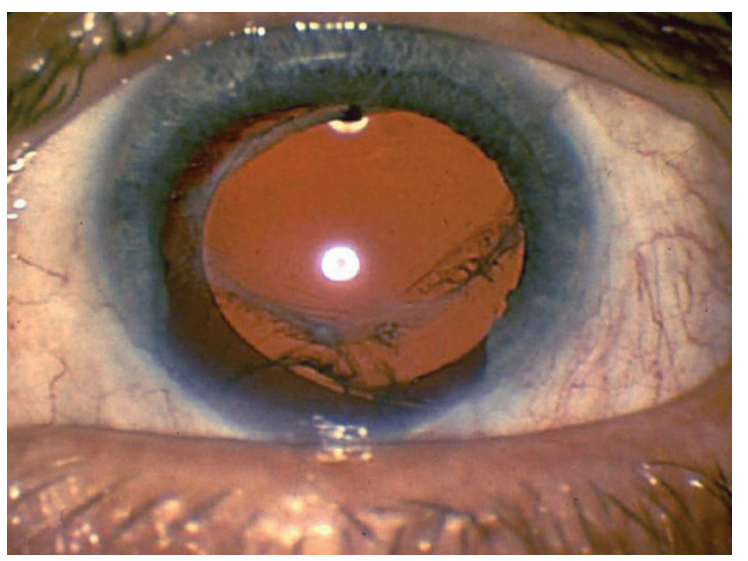

Figure Ic After phacoemulsification and intraocular lens placement, the patient achieved $20 / 30$ vision in this eye. in 1 patient $(4.5 \%)$. All patients with cataract underwent phacoemulsification surgery with intraocular lens implantation (IOL) (Figure 1c). In one case (4.5\%), nucleus drop during phacoemulsification surgery occurred, necessitating vitrectomy and nuclear fragment removal via vitrectomy and phacofragmentation. One patient developed bullous keratopathy after PLSU and subsequent phacoemulsification surgery. Of the four patients with scleral thinning, three remained stable and one underwent patch graft reinforcement because of bulging of the anterior uvea. Of the two patients with vitreous hemorrhage, one underwent vitrectomy. The other opted to wait, and the hemorrhage cleared partially in the second year after surgery. Secondary glaucoma was due to hyphema in one patient, and this patient underwent trabeculectomy. Both patients with secondary glaucoma needed antiglaucoma drops for IOP control. After PLSU, adjunctive Ru-106 plaque radiotherapy was performed in five cases with ciliary, ciliochoroidal, and iridociliochoroidal melanoma (Figure 2c).

Final Snellen visual acuity ranged from hand motions to $10 / 10$. Final $\log M A R$ visual acuity ranged from $0-1.9$ with a mean of $0.5 \log$ MAR. The mean final IOP was $16.2 \mathrm{mmHg}$ (range: $10-30 \mathrm{mmHg}$ ). We did not observe any recurrence in any patient during the follow-up period, which ranged from 1-98 months (mean: 40.1 months). Systemic metastasis was not seen in any patient with uveal melanoma at the end of the follow-up ranging from 1-98 months (mean: 45.9 months).

\section{Discussion}

The indications for exoresection have evolved over the past decades. Today many surgeons perform exoresection mainly for iridociliary and ciliary body tumors with minimal if any choroidal involvement. Endoresection, on the other hand, is usually done after proton beam irradiation or gamma-knife radiosurgery in choroidal tumors located posterior to the equator. ${ }^{13}$ The rationale for endoresection is to preserve the eye from the complications of inflammation associated with tumor necrosis.

Certain inherent difficulties of exoresection via PLSU can result in intraoperative, early (occurring $<8$ weeks after surgery), and late complications ( $>8$ weeks after surgery). One of the major intraoperative problems is the failure to dissect a scleral flap of desired thickness. It is important to find the right plane of dissection at the time the groove is made in the sclera. If the flap is not of the desired thickness initially, as judged by the lack of the underlying blue color of uvea, the plane of dissection can gently be deepened. Care should 


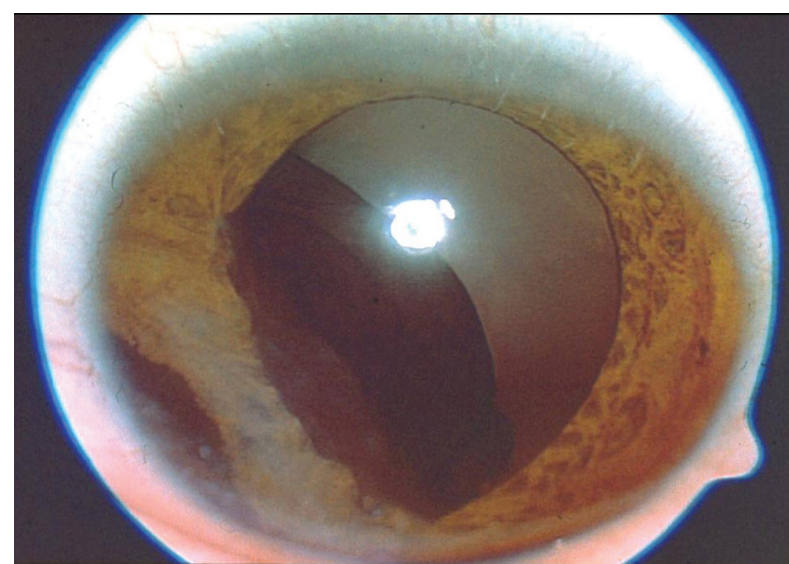

Figure 2a Massive iridociliochoroidal melanoma producing corneal touch.

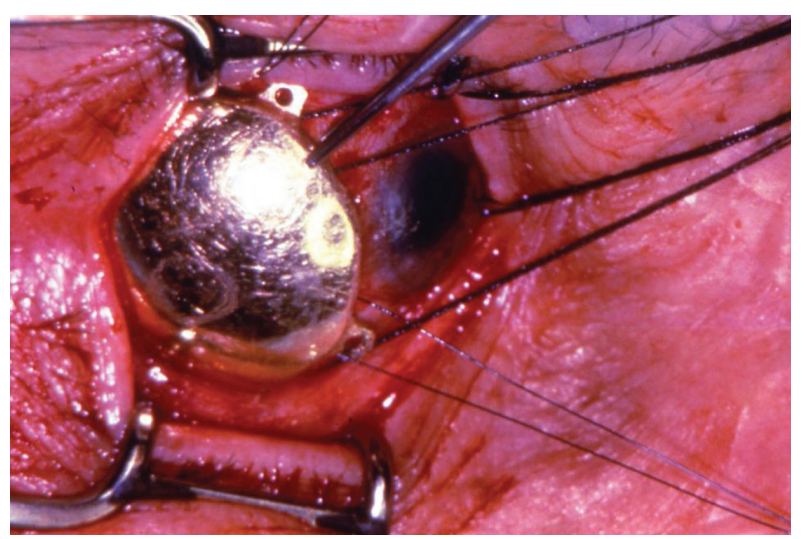

Figure 2c One month after exoresection, plaque radiotherapy with a 25 mm Ru- 106 plaque was applied to decrease the risk of postoperative recurrence.

be taken to avoid uveal perforation or vitreous loss when dissecting or deepening the flap, as this may significantly complicate the surgery.

In the senior author's experience, the second major intraoperative challenge is to open the choroidal slit and dissect the uveal tissue without damaging the underlying retina or the nonpigmented ciliary epithelium. The uveal tissue must be cauterized uniformly to decrease the chance of hemorrhage and facilitate the making of the incision in the uvea. When there is no bleeding, cutting through the uveal tissue is easier and safer in terms of not damaging the underlying retina and nonpigmented ciliary epithelium. Once a slit opening is made in the uvea using a knife needle, the surgeon should insert one blade of Vannas scissors slowly into the uveal slit, taking care to stay almost parallel to the plane of dissection. Any vertical movement of the blade may cause perforation of the retina or nonpigmented ciliary epithelium and vitreous loss.

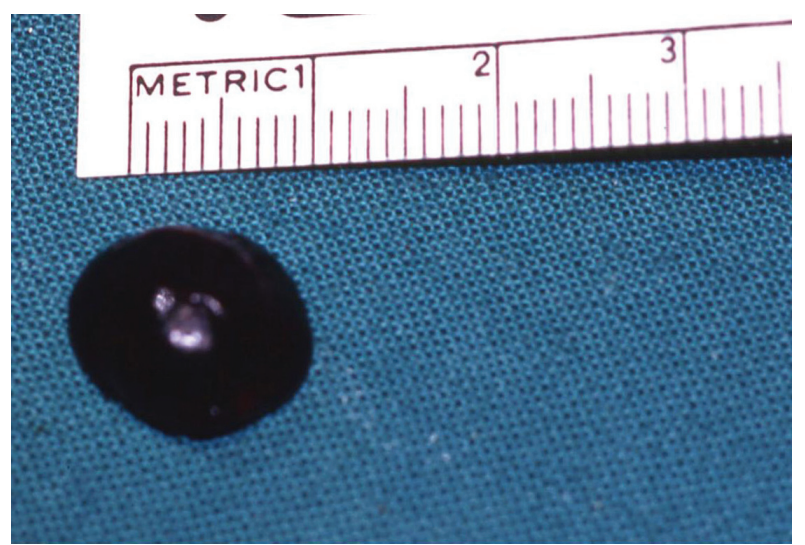

Figure $\mathbf{2} \mathbf{b}$ Gross photograph of the excised tumor showing a greater than $10 \mathrm{~mm}$ diameter uveal melanoma.

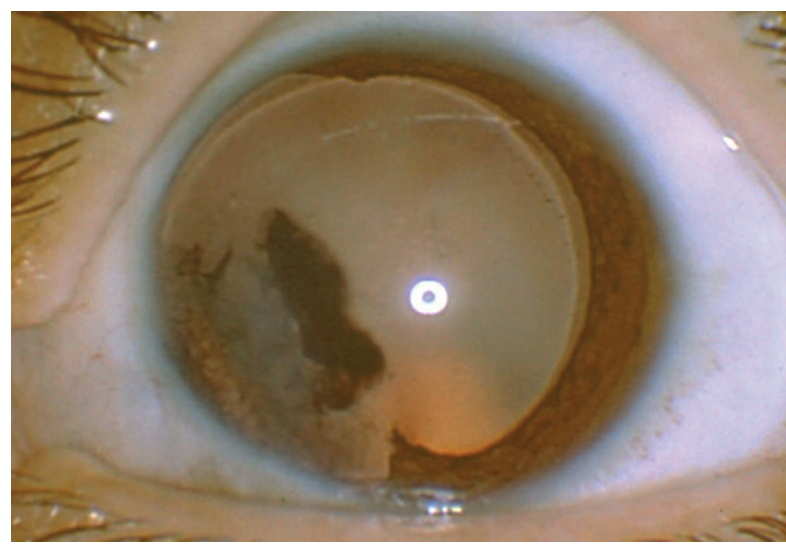

Figure 2d Anterior segment view one month after partial lamellar sclerouvectomy showing the absence of the iris from 7 to $1 \mathrm{I}$ o'clock after pupillary dilation. The corneal pigment was left untreated.

We have found that vitreous loss and subsequent vitrectomy did not lead to retinal detachment, as long as the tumor base remained anterior to the muscle insertions. We first close the scleral flap with 3-5 sutures and do vitrectomy at the wound margins subsequently. This technique is safer compared to an open sky vitrectomy approach because of the decreased risks of hypotony and possible retinal damage. Our experience with purely choroidal tumors was limited. Problems with retinal detachment may be more likely to occur in tumors located posterior to the muscle insertions.

Early postoperative complications include vitreous hemorrhage, hyphema, subretinal hemorrhage, transient blepharoptosis, cataract, retinal detachment, choroidal detachment, corneal edema, and elevated IOP. ${ }^{9-12}$ The most common early complication after PLSU surgery is vitreous hemorrhage. ${ }^{10}$ Vitreous hemorrhage was reported to occur in 28 of 95 (29\%) patients in the series by Shields and colleagues. ${ }^{10}$ It generally 


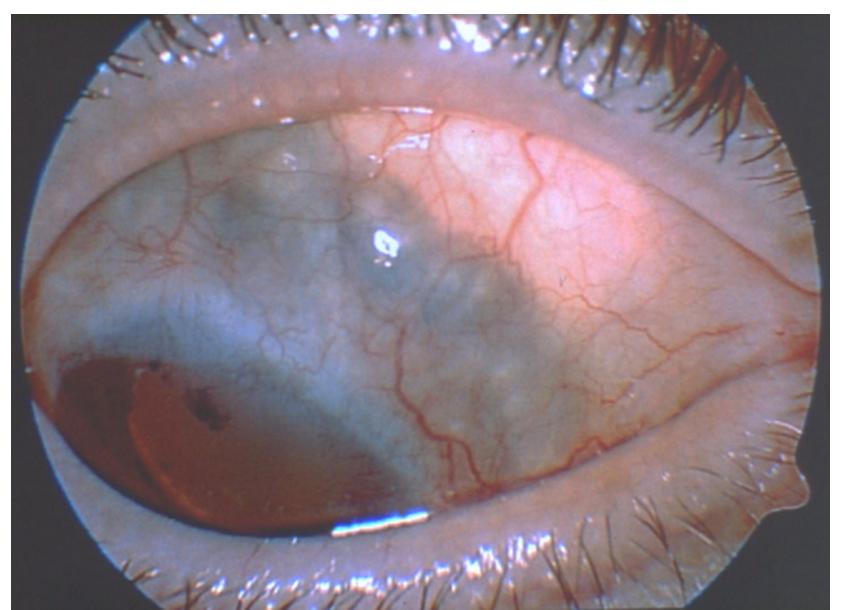

Figure 3 Scleral thinning in the superonasal quadrant of an eye that underwent partial lamellar sclerouvectomy for an iridociliary melanoma.

occurs from inadequately cauterized choroidal or ciliary body blood vessels. ${ }^{9}, 10$ In general, vitreous hemorrhage resolves spontaneously; however, when it persists beyond 8 weeks, pars plana vitrectomy can be performed. Persistent vitreous hemorrhage was seen in only 2 of $22(9 \%)$ patients in our series and in 3 of 95 (3\%) patients in the series by Shields and colleagues. ${ }^{10}$

Late postoperative complications include cataract, preretinal fibrosis, subretinal fibrosis, posterior synechiae, retinal detachment, cystoid macular edema, elevated IOP, scleral thinning and iris neovascularization. ${ }^{9-12}$ Iatrogenic lens damage can lead to cataract formation during surgery. However, often, there is a slow and progressive cataract development many months after surgery, occurring as a late complication. It is believed that if the retrolenticular vitreous is not disturbed, the risk of cataract decreases. ${ }^{9}$ The senior author has found that it is important to use a paracentesis port rather than the main corneoscleral incision for purposes of injecting viscoelastic into the anterior chamber or inflating the eye at the end of surgery. This approach poses less risk of inadvertently damaging the lens. Eleven of 22 patients $(50 \%)$ required cataract surgery with phacoemulsification and IOL implantation in our series. After PLSU, zonular defects can occur, and phacoemulsification surgery should be performed with care. Cases with more than three clock hours of zonular defects are more likely to be complicated; cataract surgery in these cases should be performed by an experienced phaco surgeon. ${ }^{14}$ Four (18\%) of our patients had scleral thinning, probably indicating a flap thickness problem, as discussed previously. Usually, this problem can be followed with observation unless marked bulging of the uveal tract develops, in which case scleral reinforcement with a patch graft should be considered.

Tumor recurrence may follow incomplete removal of malignant tumors with positive surgical margins. To avoid this serious problem, adjunctive plaque radiotherapy has been suggested. In the series by Damato and colleagues, adjunctive plaque radiotherapy was shown to be effective for decreasing local tumor recurrence after PLSU. ${ }^{15}$ Histopathologic study of enucleated eyes harboring melanoma show melanoma cells in the sclera in $56 \%$ of cases. ${ }^{16}$ The presence of untreated intrascleral melanoma cells is another reason to perform plaque radiotherapy after PLSU. ${ }^{14}$ Kim and colleagues speculated that dissemination of melanoma cells during PLSU may account for occurrence of noncontiguous new tumor after surgery. ${ }^{17}$ We performed plaque radiotherapy using Ru-106 plaques in five cases with ciliary, ciliochoroidal, and iridociliochoroidal melanoma.

Distant metastasis presents a challenging problem in patients with uveal melanoma. In the series by Damato and colleagues, predictors for metastatic disease included: epithelioid/mixed cell type; largest basal tumor diameter $\geq 16 \mathrm{~mm}$; patient age greater than 60 years; secondary enucleation for gross residual or recurrent tumor; secondary enucleation for extraocular extension; and superior tumor location. ${ }^{18}$ Memmem and McLean found that epithelioid/mixed tumor cell type and involvement of the surgical margin by the melanoma were risk factors for metastasis. ${ }^{19}$ In the series by Shields and colleagues, 5 of 95 patients $(7 \%)$ developed metastatic disease, whereas in the series by Damato and colleagues, 52 of 332 patients (15\%) had metastatic disease. ${ }^{10,18}$ Bechrakis and colleagues and Augsburger and colleagues found no statistically significant difference in the survival rate of patients treated with PLSU versus those treated with plaque radiotherapy ${ }^{20,21}$ Furthermore, visual outcomes appeared to be better after PLSU than after radiation treatment. ${ }^{21,22}$ At a mean follow up of 46 months, we did not record any metastasis in our series of 16 patients with uveal melanoma.

Our results show that the complication and recurrence rates at short term were comparable to the results in series published by experienced authors. Although the surgical technique poses some difficulties, exoresection seems to be an effective treatment option in selected cases of iridociliary and ciliary body tumors. In ciliary body tumors extending posteriorly into the choroid, separation of the retina from the tumor can be difficult. Therefore, it is advisable to limit the use of exoresection to tumors located anterior to the ora serrata especially for inexperienced surgeons. The purpose of this paper has been to describe potential surgical 
problems that might be encountered during exoresection and methods to avoid and/or manage them. Ocular oncologists with limited training in exoresection should not be reluctant to do this type of surgery, provided they understand the basic surgical principles involved and think they are able to do it. The surgery is inevitably associated with several postoperative complications requiring frequent patient monitoring.

\section{Disclosures}

The authors report no conflicts of interest in this work.

\section{References}

1. Shields CL, Manquez ME, Ehya H, Mashayekhi A, Danzig CJ, Shields JA. Fine-needle aspiration biopsy of iris tumors in 100 consecutive cases: technique and complications. Ophthalmology. 2006;113:2080-2086.

2. Marigo FA, Finger PT. Anterior segment tumors: current concepts and innovations. Surv Ophthalmol. 2003;48:569-593.

3. Grossniklaus H. Size is a sign of malignancy in melanocytic lesions of the iris. Ocular Surg News. 2008;19:12.

4. Biswas J, Kumar SK, Gopal L, Bhende MP. Leiomyoma of the ciliary body extending to the anterior chamber: clinicopathologic and ultrasound biomicroscopic correlation. Surv Ophthalmol. 2000;44:336-342.

5. Fineman MS, Eagle RC Jr, Shields JA, Shields CL, De Potter P. Melanocytomalytic glaucoma in eyes with necrotic iris melanocytoma. Ophthalmology. 1998;105:492-496.

6. Shields CL, Shields JA, De Potter P, Singh AD, Hernandez C, Brady LW. Treatment of non-resectable malignant iris tumors with custom designed plaque radiotherapy. Br J Ophthalmol. 1995;79:306-312.

7. Shields CL, Naseripour M, Shields JA, Freire J, Cater J. Custom-designed plaque radiotherapy for nonresectable iris melanoma in 38 patients: tumor control and ocular complications. Am J Ophthalmol. 2003;135:648-656.

8. Damato B, Woulds WS. Indications for trans-scleral local resection of uveal melanoma. Br J Ophthalmol. 1996;80:1029-1030.
9. Shields JA, Shields CL. Surgical approach to lamellar sclerouvectomy for posterior uveal melanomas: The 1986 Schoenberg Lecture. Ophtalmic Surg. 1998;19:774-780.

10. Shields JA, Shields CL, Shah P, Sivalingam V. Partial lamellar sclerouvectomy for ciliary body and choroidal tumors. Ophthalmology. 1991;98:971-983.

11. Damato BE, Paul J, Foulds WS. Risk factors for residual and recurrent uveal melanoma after trans-scleral local resection. Br J Ophthalmol. 1996;80:102-108.

12. Damato BE, Paul J, Foulds WS. Predictive factors of visual outcome after local resection of choroidal melanoma. Br J Ophthalmol. 1993; 77:616-623.

13. Bechrakis NE, Foerster MH. Neoadjuvant proton beam radiotherapy combined with subsequent endoresection of choroidal melanomas. Int Ophthalmol Clin. 2006;46:95-107.

14. Lindquist TD. Capsulorhexis, phacoemulsification, and posterior chamber lens placement following iridocyclectomy. Ophthalmic Surg. 1992;23:44-46.

15. Damato B. Adjunctive plaque radiotherapy after local resection of uveal melanoma. Front Radiat Ther Oncol. 1997;30:123-132.

16. Histopathologic characteristics of uveal melanomas in eyes enucleated from the Collaborative Ocular Melanoma Study. COMS report no. 6. Am J Ophthalmol. 1998;125:745-766.

17. Kim JW, Damato BE, Hiscott P. Noncontiguous tumor recurrence of posterior uveal melanoma after transscleral local resection. Arch Ophthalmol. 2002;120:1659-1664.

18. Damato BE, Paul J, Foulds WS. Risk factors for metastatic uveal melanoma after trans-scleral local resection. Br J Ophthalmol. 1996; 80:109-116.

19. Memmen JE, McLean IW. The long-term outcome of patients undergoing iridocyclectomy. Ophthalmology. 1990;97:429-432.

20. Bechrakis NE, Bornfeld N, Zöller I, Foerster MH. Iodine 125 plaque brachytherapy versus transscleral tumor resection in the treatment of large uveal melanomas. Ophthalmology. 2002;109:1855-1861.

21. Augsburger JJ, Lauritzen K, Gamel JW, et al. Matched group study of surgical resection versus cobalt-60 plaque radiotherapy for primary choroidal or ciliary body melanoma. Ophthalmic Surg. 1990;21:682-688.

22. Char DH, Miller T, Crawford JB. Uveal tumour resection. Br J Ophthalmol. 2001;85:1213-1219.
Clinical Ophthalmology

\section{Publish your work in this journal}

Clinical Ophthalmology is an international, peer-reviewed journal covering all subspecialties within ophthalmology. Key topics include: Optometry; Visual science; Pharmacology and drug therapy in eye diseases; Basic Sciences; Primary and Secondary eye care; Patient Safety and Quality of Care Improvements. This journal is indexed on

Submit your manuscript here: http://www.dovepress.com/clinical-ophthalmology-journal

\section{Dovepress}

PubMed Central and CAS, and is the official journal of The Society of Clinical Ophthalmology (SCO). The manuscript management system is completely online and includes a very quick and fair peer-review system, which is all easy to use. Visit http://www.dovepress.com/ testimonials.php to read real quotes from published authors. 